\title{
Ueber Molorchus abbreviatus Panz. und Salicis Muls.
}

von

\author{
Oberlieutenant L. v. Heyden.
}

(Hierzu Taf. IV. Fig. 6, 7.)

D urch die Bereitwilligkeit, mit welcher meine beiden Freunde, die Herren Stern in Frankfurt, mir ihr sämmtliches Material über diese Arten, welche sie in Anzahl zogen, zur Untersuchung anvertrauten, bin ich in den Stand gesetzt, über die Verwandtschaft und Unterschiede beider einen genügenden Aufschlufs zu geben.

Vor allem mufs hervorgehoben werden, dafs es zwei gut unterschiedene Arten unter den grofsen Molorchen giebt. Linné war der erste, welcher einen grofsen Molorchus unter dem Namen major beschrieb. Seine Diagnose ist aber so kurz, dafs nur zweifelhaft angegeben werden kann, welche der beiden bis jetzt hekannten Arten er darunter verstanden hat. Fast sämmtliche späteren Schriftsteller schreiben einfach die Linné'sche Diagnose: elytris dimidiutis, ferrugineis, immaculatis, antennis brevibus nach; zu diesen gehört auch Fabricius, der, wie mir scheint, ohne Veranlassung den Linnéschen Namen major in abbreviatus abänderte. Im System. Entomol., seinem ältesten Werk über Käfer, führt er zum ersten Male den Namen abbreviatus an, giebt aber dazu die unveränderte Linné'sche Diagnose, wie in allen seinen späteren Werken. Linné sagt aber in der kurzen Beschreibung (Fauna Suec. No. 838.) . ... antennae nigrae, basi ferrugineae, .... abdomen longiusculum, lineare, nigrum; es sind dies Angaben, welche nur auf $\boldsymbol{M}$. Salicis б우 passen. Panzer giebt gleichfalls die Linné'sche Diagnose, aber mit dem Fabricius'schen Namen; sein Thier ist sicher nicht Salicis, sondern die andere robustere Art, die zum ersten Male von ibm sehr kenntlich abgebildet wird. Dieser Art gebührt also der Panzer'sche Name abbreviatus. - M. Salicis Muls. ist die kleinere Art 
mit schwarzen Knieen, zu der ich fraglich den major L. stelle. Büttner's Populi ist sicher nach kleinen $\delta$ von Salicis aufgestellt. Mulsant war der erste, welcher aus dem major eine eigene Art unter dem Namen Salicis ausschied und kenntlich beschrieb. Mulsant's Name verdient also den Vorzug, obgleich eine grofse Anzahl Molorchen älterer Schriftsteller, alle unter dem Linné'schen Namen major, darauf bezogen werden müssen.

Molorchus abbreviatus lebt in Eichen und Buchen (v. Heyden, Stern), Ulme, Maulbeerbaum (Mulsant), Pappel (Mulsant) [? wenn keine Verwechselung mit Salicis vorliegt, denn diese Angabe rührt von Dupont her].

M. Salicis Muls. lebt in Weiden (v. Heyden, Stern, Redtenbacher), Aspen (Büttner, Stern).

Die Männchen beider Arten unterscheiden sich von ihren Weibchen durch die Form des letzten Hinterleibssegmentes; bei den ersteren ist dieses verhältnifsmäfsig klein und das vorletzte oben sehr stark gewölbt; bei den Weibchen ist das letzte Glied sehr lang, ziemlich gleichbreit, nach hinten nur allmälig verschmälert, dabei viel flacher. Bei den Männchen sind die Fühler nach der Spitze zu, vom 5ten Gliede anfangend, gewöhnlich dunkler.

1) Molorchus abbreviatus Panz. ist gewöhnlich die grössere der beiden Arten: das gröfste mir bekannte $\sigma^{-7}$ hat $13 \frac{1}{2}$ Linien, das gröfste \& $14_{2}^{1}$ Linien. Die Fühler sind stärker und robuster gebaut wie bei Salicis, beim $q$ hellbraun, beim $\sigma^{\top}$ dunkelbraun, an der Spitze am dunkelsten. Das Ha'sschild ist beinahe so breit wie lang, der ganze Vorder- und Hinterrand, sowie die Seiten, sind "sehr" stark goldglänzend behaart, so dafs an diesen Stellen kaum die schwarze Grundfarbe zu erkennen ist. Die Brust ist in beiden Geschlechtern ebenfalls sehr stark goldglänzend behaart. Die Flügeldecken sind hinten fein schwarz gerandet. Die Innenseite der Mittelschenkel hat in beiden Geschlechtern stets einen schwarzen, mehr oder weniger starken Schatten. Die schwarze Binde der Hinterschenkel endigt ziemlich weit ,vor" den gelben Knieen (bei Salicis sind die Kniee stets schwarz); oft ist sie auf der Unterseite kaum durch etwas dunklere Färbung angedeutet; ein gleicher schwarzer Fleck findet sich stets, mehr oder weniger deutlich, auf der Ober- und Aufsenseite der Endspitze der Hinterschienen. Bei den $\sigma^{\top}$ sind das 1ste, 2te, 3te Hinterleibssegment auf der Unterseite gelbbraun mit schwarzem Schatten in der Mitte des ersten Segments; der Rest des Hinterleibs ist schwarz. Beim 오 sind sämmtliche Segmente auf der Unterseite hinten gelbbraun, an den ersten am brei- 
testen, bei einem Exemplare auch das ganze 1ste und 2te Segment auf der Unterseite, mit dunklerem Schatten auf der Mitte und an den Seiten. Die Oberseite der drei ersten Segmente ist stets ganz gelbbraun. Beim $\sigma^{\top}$ ist das vorletzte Segment kugelig gewölbt, das letzte unter dem vorletzten ziemlich stark verborgen, viel schmäler, abschüssig, an den gerandeten Seiten und hinten abgerundet; oben in der Mitte befindet sich eine kurze, sehr deutliche Falte. Das vorletzte (beim ठ) auf der Unterseite sehr stark vertieft, von zwei scharfen, schräg nach vorne gehenden Falten eingefafst. Beim 우 ist das vorletzte Segment von den andern wenig an Form verschieden, das letzte ebenso lang wie das vorletzte, nicht darunter verborgen, wenig gewölbt, an der Basis etwas schmäler als das vorletzte, die abgerundete Spitze halb so breit als vorn, an den Seiten, besonders in der Mitte, wulstig gerandet, daneben mit einem länglichen Eindruck.

2) Molorchus salicis Muls. ist durchschnittlich kleiner ( $\sigma^{\prime}$

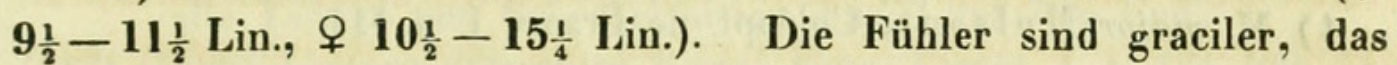
Halsschild schmäler, viel länger als breit, nur an den Seiten mit goldglänzenden Haaren besetzt, die an den vier Ecken dichter beisammen stehen. Die Flügeldecken sind hinten gleichfarbig, oder mit elwas dunklerem Fleck an der Spitze. Die Brust ist schwächer behaart. Der schwarze Fleck an der Innenseite der Mittelschenkel ist nie vorhanden, ebenso fehlt stets der schwarze Fleck an den Hinterschienen. Der glänzend schwarze Fleck der Hinterschenkel nimmt die ganze Schenkelverdickung ein und reicht hinten bis an die Schienen. Die weiblichen Fühler sind gelbbraun mit dunklerer Spitze; die männlichen schwärzlich, das 1-4 Basalglied und die Wurzel des 5ten rothbraun. Bei dem $\sigma$ sind auf der Unterseite die drei ersten Hinterleibsringe gelbbraun, der dritte mehr oder weniger gebräunt; bei den $ᄋ$ ist der Hinterleib ganz schwarz, und nur die Seiten der zwei ersten Segmente sind oben gelbbraun. Die beiden letzten Segmente des $\sigma$ sind oben ebenso wie bei abbreviatus, nur fehlt das sehr deutliche Fältchen der Oberseite. Die Unterseite des vorletzten Segments ist an der Spitze nur schwach mondförmig vertieft. Beim $q$ ist das vorletzte Segment wenig von den ersten verschieden, das letzte so lang wie das vorletzte, schmal, ziemlich gleichbreit, hinten zugerundet, an den Seiten wie bei major gewulstet und vertieft. Das letate Drittel der Flügel ist besonders beim $q$ schwärzlich, bei abbreviatus befindet sich nur ein tiefschwarzer, nach aufsen mehr oder weniger verwaschener Fleck am Spitzenaufsenrand. Herr Stern besitzt 
eine salicis var. $\sigma^{\prime}$, bei der die ganzen Hinterschenkel nur einen sehr schwachen Schein von schwarzer Färbung haben.

Zu 1. Molorchus abbreviatus Panzer gehören folgende Citate:

1) M. abbreviatus Panz. Faun. German. XLI. 20. (nur die Abbildung ist sicher).

2) ?M. abbreviatus Panz. Deutschl. Insektenfauna p. 280.

3) M. major Guérin Iconograph. II. pl. 44, 8. scheint wegen der derben Fühler und des kurzen breiten Halsschilds sicher hierher zu gehören. Dagegen sprechen zwar die schwarzen Kniee, die ganze Figur ist aber flüchtig gemalt.

a. (1840) Castel nau, Hist. natur. des Ins. coléopt. T. II. p. 442. 1. pl. 50. fig. 4. als M. major (schlechte Abbildung, aber wegen des kurzen Halsschildes und der dicken Fühler sicher hierher; desgl. nach Beschreibung).

b. (1843) Necydalis major Boitard Nouv. Manuel complet d'Entomol. T. II. p. 287.

1) M. major Mulsant, Coléopt. de France I. p. 111.

Zu 2. Molorchus salicis Muls. gehört:

?1) (1761) Necydalis major L. Fauna suec. ed. II. No. 838.

?2) (1767) - - L. Syst. nat. ed. XII. T. I. pars II. p. 641. 1.

?3) (1788-93) - - L. Syst. nat. ed. Gmelin p. 1878.

?4) (1753) Musca cerambyx major S chäffer, der Afterholzbock p. 512. f. 1-5. scheint ein grofses 오 von salicis. Die Farbenangaben in den Abbildungen sind ungenau.

5) (1764) Necydalis primus Schäffer lconogr. t. 10. f. 10. 11. Schäffer benannte die Arten nicht, sondern numerirte sie in den Gattungen. Seine Abbildung läfst an der zierlichen Gestalt des Körpers und der Fühler und den schwarzen Knieen sehr deutlich den salicis erkennen. Die vortreffliche Abbildung stellt ein 오 dar, mit dem charakteristischen schwarzen Hinterleib mit gelben Seiten.

?6) (1764) Gronovius Enumer. Insect. p. 173 No. 626. t. 15. (ohne Namen). Linné'sche Diagnose; nach Abbildung $\sigma^{T}$.

?7) (1781) Necydalis ichneumonea De Geer (Geschichte der Insekten V. p. 314. 1) gehört nach Abbildung (sehr deutlich die schwarzen Kniee) und Beschreibung zu salicis. Die ganze Abbildung fig. 1. stellt ganz richtig ein 우 vor; der abgebildete Hinterleib fig. 2. gehört, wie angegeben, zu einem $\sigma$.

? 8) (1775) Leptura abbreviata Fabr. Syst. Ent. p. 199. 18.

? 9) (1781) - - Fabr. Spec. Ins. I. p. 250. No. 27.

? 10) (1787) - - Fabr. Mlantissa 1. p. 160. No. 35.

?11) (1792) Molorchus abbreviatus F abr. Ent. Syst. I. II. p. 356.

? 12) (1801) - - Fabr. Syst. Eleuth. II. p. 374. 
13) (1784) Neeydalis major Laicharting (Beschreib. d. Tyrol. Ins. II. p. 173. 1.) gehört nach der Beschreibung sicher zu salicis, und zwar ist es nach der Hinterleibsfarbe ein $\delta$.

14) (1784) Necydalis major Harrer Beschreibungen etc. zu Schäffer Iconograph. p. 241. No. 402.

15) (1789) Necydalis major Villers Entom. I. p. 277. Nach Beschreibung $\left(\sigma^{\top}\right.$ 은, besonders der Hinterleibsfarbe (maris abdomen ferrugineum, apice nigrum, feminae nigrum).

16) (1789-1808) Necydalis major Oliv. (Ent. IV. No. 74. p. 5. t. 1. fig. 1 a. b.) gehört nach Beschreibung .... antennae nigrae, basi ferrugineae .... abdomen nigrum .... Corselet presque cylindrique .... sicher hierher; ebenso nach der Abbildung, besonders der Gröfse des Körpers, dem langen Halsschild und dem bis auf das 1ste Segment schwarzen Hinterleib (Fig. I.b stellt ein 오 vor; Olivier giebt dies zwar' nicht an, aber es ist leicht an der Hinterleibsform zu erkennen). Die Spitzen der Hintertarsen sind zwar schwarz; es kann dies aber vermalt sein, da alle Tarsen- und Schenkelspitzen dunkel gehalten sind.

? 17) (1790) Leptura abbreviata Rossi Faun. Etrusc. I. p. 164. 409.

? 18) (1794) - Rossi Faun. Etrusc. ed. Hellwig T. I p. 186.409.

? 19) (1798) Gymnopterion majus Schrank Faun. boic. I. p. 688 scheint nach der kurzen Beschreibung: Gröfse von 11 Linien und dem schwarzen Punkt am Ende der Decken, hierher zu gehören.

?20) (1800) Mol. major Pa yk. Faun Suec.III. p. 129 (gute Beschreibung).

?21) (1805) Necydalis major Bechstein u. Scharfenberg Forstins.

I. p. 220. 1. ist salicis 오 nach der Angabe: am Ende der Flügeldecken manchmal ein schwarzer Punkt, nach dem schwarzen Hinterleib und den einfärbig rothbraunen Fühlern; nur pafst nicht: Hinterfülse schwarz.

22) (1819) Mol. populi Büttner Germar Ent. 3. p. 245. No. 12.

23) (1827) Mol. abbreviatus Gyllenh. Ins. Suec. 4. p. 120. ð오.

24) (18:39) Mol. salicis Mulsant Coléopt. de France I. p. 112. No. 2. pl. 4. fig. f.

Mol. major Redtenb. Fauna austr. ed. II. p. 856.

25) (1843) Necyd. salicis (Dup.) Boitard 1. c p. 287. Die Boitardschen Beschreibungen sind kurz, aber lassen beide Arten ( $a b$ breviata und salicis) genügend erkennen.

26) (1856) Mol. major B a ch Käferfauna pag. 26. 1. Bach vermengt beide Arten, nach der Angabe der Lebensweise.

27) (1859) Mol. major Bose Käfer Deutschl. p. 580. 1. 


\section{$2 \mathrm{BHL}$ Biodiversity Heritage Library}

Heyden, Lucas von. 1864. "Ueber Molorchus abbreviatus Panz. und Salicis Muls." Berliner entomologische Zeitschrift / herausgegeben von dem Entomologischen Vereine in Berlin 8(304)329-333. https://doi.org/10.1002/mmnd.18640080311.

View This Item Online: $\underline{\text { https://www.biodiversitylibrary.org/item/36390 }}$ DOI: https://doi.org/10.1002/mmnd.18640080311

Permalink: https://www.biodiversitylibrary.org/partpdf/210025

\section{Holding Institution}

Smithsonian Libraries

\section{Sponsored by}

Smithsonian

\section{Copyright \& Reuse}

Copyright Status: Public domain. The BHL considers that this work is no longer under copyright protection.

This document was created from content at the Biodiversity Heritage Library, the world's largest open access digital library for biodiversity literature and archives. Visit BHL at https://www.biodiversitylibrary.org. 